\section{A LARGE ASTRONOMICAL TELESCOPE FOR GREAT BRITAIN}

A DISCUSSION on the use of a large telescope in Britain took place at a meeting of the Royal Astronomical Society on June 14. This discussion had its origin in the presidential address delivered to the Society earlier the year by Prof. H. H. Plaskett, who pofint out that, lacking a telescope of large light-gdy fring power, British contributions to a large pape of observational astronomy would of necessity pome practically negligible. He also emphasjzd the interrelation between observational and the Hetical prik, and reminded the Society how in frast tapatance of physics has depended upon obforvdtion discoveries in astronomy. $\mathrm{H}_{\theta}$ put forward suggestions for the construction of an instru= ment of the Schmidt type with a mirror of 74 in. aperture, suitable both for direct photography and for slit spectroscopy. The estimated cost of such an instrument, with proper ancillary equipment, is $£ 100,000$, and Prof. Plaskett suggested that the Society should take the initiative in making application for the necessary capital cost and running expenses, the idea being that the equipment should be installed at a suitable central site and should be available for use by all the observatories in the United Kingdom.

During the four months following Prof. Plaskett's address, British astronomers gave his proposal active consideration, and a crowded meeting at Burlington House on June 14 heard the scheme fully and freely discussed. In the absence abroad of the Astronomer Royal, Sir Harold Spencer Jones, his opening contribution on observing conditions in Great Britain was read by Dr. R. d'E. Atkinson. Sir Harold pointed out that estimates of night cloudiness at a particular site must, in the absence of direct data, be made by rather roundabout methods. At Greenwich, night sky records have been made by obtaining trails of circumpolar stars with a fixed camera, and there is a general positive correlation between the yearly totals of clear sky obtained from these records and the annual sunshine totals. Sunshine records indicate that the south-east is the most suitable region in England for astronomical observations. On the basis of the sunshine records for Herstmonceux Castle (the new site chosen for the Royal Greenwich Observatory), Sir Harold predicted that for this site 1,500 hours of clear dark sky should be obtained in an average year.

The question before us, then, is whether this total of $\mathbf{1}, 500$ hours is enough to justify the installation of a large telescope. Sir Harold pointed out that only a small fraction of the astronomer's time is spent in actual telescope work. For most observations by far the larger part of the total work is taken up by plate measurement, reduction of the measures and analysis of the raw material thus obtained. For a long-term, fairly routine programme, such as stellar parallax work, the ratio of observing to non-observing time is found by experience to be about 1 to 8 . In such work only a few stars are measured on each plate; but for positional work, such as zone photography with a wide-angle lens, hundreds of stars are measured on a single plate, and heavy computations are involved; for this work the ratio may reach 1 to 700 . Between these two extremes lie ratios applicable to other programmes, and as a rough average the office time required can be put down as a hundred times the observing time. With this ratio, an annual total of 1,500 hours observing time would keep a considerable number of astronomers fully occupied, a conclusion which is confirmed by experience with the 72-in. reflector at Victoria, B.C., "where the annual total of clear night sky is less than 1,500 hours. From the magnificent results obtained there on a variety of well-planned programmes, Sir Harold deduced that the installation of a large telescope in southern England would be fully justified. This conclusion was of great interest to the meeting, since some rather defeatist statements have been made in the past to the effect that the British climate is such that no adequate return can be expected from the erection of a large telescope.

The discussion was continued by Prof. W. M. H. Greaves, Astronomer Royal for Scotland, who said that it was his task to outline the programmes for which the proposed new equipment could be used. $\mathrm{He}$ proposed to fulfil this task by asking a rather wider question: What contributions can British astronomers make to the major problems of astronomy, given the requisite equipment ? Refusing to subscribe to the view that we should be solely concerned with the provision of a large telescope in Great Britain, he said that the major problems demand observational material from both hemispheres, and in the present state of the world it is largely for the countries of the northern hemisphere to make adequate provision for work in southern latitudes. British astronomers cannot escape their responsibilities in this direction, especially as the British Commonwealth includes southern countries with good climatic conditions. How, then, are our responsibilities being met as regards the major problems of astronomy, and what can be done given the proposed new equipment?

One major research is the study of the structure and dynamics of the galaxy, and this requires photo. metric work combined with good proper motions, parallaxes and radial velocities. As regards proper motions and parallaxes, we are already playing our part, and we can be confident that the Greenwich and Cape Observatories would see to it that we continue to do so. As regards radial velocity work, the proposed programme with the 74-inch telescope at the Radeliffe Observatory, Pretoria, will complete the magnificent work done in the northern hemisphere at Victoria, and the two series will constitute an achievement of which British astronomers can be proud. But galactic research is primarily based on photometric work; the main observational raw material consists of counts of stars down to assigned apparent magnitudes in various fields of the sky, and the evaluation of the absorption of light by inter. stellar material is a photometric problem. The necessary photometric programmes consist of $(a)$ a large-scale survey of areas distributed over the whole sky, which would lead to a general smoothed picture of the galactic system; and (b) a more detailed survey based on the study of areas selected for the enhanced presence or absence of obscuring material. It is essential that the photometry should be of first-class quality; there is no room in this research for poor quality mass-production.

The British contribution to this very important photometric work is, to our shame, practically zero at present. As regards $(a)$, the Mount Wilson survey can be said to have met the immediate requirements in the northern hemisphere, but there remains (b), where we could play an important part if we had a. 
large telescope of the Schmidt type. When we come to consider the state of the southern hemisphere work, the sad fact emerges that, so far as photometry of precision is concerned, astronomy has practically the whole way to go in both $(a)$ and $(b)$. Here is a chance for redeeming our past neglect in one of the major problems of astronomy. Prof. Greaves believes that in determining our contribution we should give priority to the erection of a Schmidt telescope in the southern hemisphere, to be operated by British astronomers. But northern work is very important; we should play our part by erecting a large Schmidt telescope in Great Britain as well.

Another major problem is the study of the physics of individual stars and other celestial objects. In this comparatively young and rapidly developing subject, the main observational weapon is the spectroscope. But to obtain spectra of reasonable dispersion, one must feed the spectroscope with sufficient light, and at present we are not playing our part mainly because our instruments are too small. By the imminent erection of the 74-in. reflector with spectroscope at Pretoria we could claim that we are playing our part in southern work, but observations in both hemispheres are necessary. To maintain the necessary close association of astrophysics with the main body of physical science under present conditions, we must push on with observational astrophysies, including the development of new methods, in Great Britain. But the tendency for clear sky to occur in comparatively short spells in the British Isles is emphatically against any programme involving long exposures, and to obtain material of real value a large telescope is a necessity. Prof. Greaves commented that if anyone wished. to carry out any particular astrophysical programme in both hemispheres, and if he only had funds for, say, a 72-in. and a 36-in. reflector, then common sense would dictate that the larger instrument should be erected in Britain, and the smaller in a country like South Africa where the climate is suitable for longexposure work. The two parts of the programme would then keep in step. As it is, unless the proposed equipment is fortheoming, he believes that we in Great Britain will lag behind in observational astrophysics. There is plenty of work to be done, but a large telescope is a necessity if we are to take part in it.

With regard to the cosmological study of the universe of galaxies, which constitutes a third major research, Prof. Greaves said that at present we are contributing precisely nothing. Good photometry can discriminate between some of the rival hypotheses that have been advanced, but so far the very valuable pioneer work that has been done does not come up to the requisite standard of photometric precision; given a large Schmidt instrument we could play a notable part. $\mathrm{He}$ concluded by saying that in all three of the major problems considered, we could contribute materially given the necessary equipment, but that without such equipment our contributions would remain practically ineffective and British observational astronomy would slowly die. The connexion between observational and theoretical astronomy is so intimate that the latter could not long survive the death of the former; and the death of British astronomy could only inflict harm on the main body of physical science.

The meeting then considered details of the proposed instrument. Mr. F. J. Hargreaves dealt with the mechanical problems involved in the design of a large telescope. Not all mountings, as he pointed out, allow all parts of the sky to be reached, and the fact that the mirror alone of a 72-in. reflector would weigh about two tons means that the engineer is set many problems in avoiding flexure. With the aid of diagrams, Mr. Hargreaves described the two main classes of mounting: the 'overhung' type, in which a declination axis, set at right angles to the polar axis (about which the instrument turns), carries the main tube at one end balanced by a counterweight at the other ; and the 'trunnion' type, in which the tube is slung within the polar axis and is thus supported from both sides, a counterpoise being unnecessary. Each has its advantages and drawbacks, and Mr. Hargreaves described some possible ways of combining the good points of each without their disadvantages.

A desirable feature of a modern telescope, incorporated in the new 74-in. Radcliffe reflector, is an optical laboratory, below the dome floor, into which the light is directed by auxiliary reflectors. This avoids the need for carrying heavy or awkwardly shaped apparatus on the telescope itself, and enables the observer to work in comfort in controllable conditions. With the overhung construction modified as at Pretoria, this can be achieved with only two additional reflexions, but with the conventional trunnion mounting four are needed. Mr. Hargreaves suggested a modification of his own which eliminates two of these, and concluded by showing how this could be combined with many of the good mechanical features of other existing mountings to give a design for the proposed new telescope which could be both mechanically sound and optically efficient.

In a contribution concerned entirely with arriving at the most desirable optical train for the telescope, Dr. E. H. Linfoot pointed out that the classical Cassegrain can scarcely be bettered as a condensing system for stellar spectroscopy, but it has a very limited field. A better system for astrographic work is the Schmidt camera, in which the aberrations of a spherical mirror are removed by a figured plate at its centre of curvature. Speaking as an optician with an interest in astronomy, Dr. Linfoot recommended a Sohmidt telescope of aperture ratio about $f / 3 \cdot 5$ and focal length 14 or $15 \mathrm{ft}$. (stop diameter 50 in. for an unvignetted field of $7^{\circ}$ ), convertible into a 72 -in. spectrograph condenser by the addition of a small auxiliary convex mirror.

Dr. C. R. Burch, in agreeing with Dr. Linfoot's conclusions, appealed for a design which would not be determined by any cheese-paring austerity considerations. Quite apart from the astronomical value of a large telescope in Great Britain, its design and construction would, in his view, stimulate and inspire the technical opticians and the optical industry to an extent which would in large measure justify the cost. Astronomical optics has already provided now ideas for use in microscopy, for example, and there is no reason to believe that the fountain of good ideas has run dry.

To add point to this last remark, Mr. W. J. Bates then described a new device for testing mirrors which he has called a "wave-front shearing interferometer'. Two views of the wave-front coming from the mirror are formed by division at a half-silvered surface, and are recombined with a relative displacement or shear. Interference fringes are thereby formed which are analogous to the contact fringes obtained by moving a test-plate of the same asphericity over the mirror under test. The method eliminates 
the usual comparison beam necessitating a worked surface comparable in dimensions with that to be tested, and the apparatus (which was exhibited) in its present undeveloped state occupies about a sixinch cube. It might therefore be attached permanently to the telescope for frequent tests under working conditions.

Dr. W. M. Hampton, technical director of Messrs. Chance Bros. and Co., Ltd., next spoke on the construction of the mirror. As a material capable of taking a high polish, with a hard permanent surface and low thermal expansion, he said, glass is unrivalled. No technical difficulties would arise in the production of a 72-in. blank in Great Britain, though there would be serious commercial ones. A supply of suitable glass already exists ; the difficulty would be in the provision of facilities and especially space for transferring it from the furnace to the moulds and annealing kilns. Dr. Hampton emphas. ized, however, that his remarks applied to the glass for the main mirror, where its function is to provide an accurately figured surface. A Schmidt plate presents a very different problem, since the highest optical homogeneity is required for this, and a lowexpansion glass has never yet been produced in the requisite optical quality.

The meeting then proceeded to more open informal discussion. A question from Prof. Greaves elicited the fact that, should an instrument of longer focal length than the $15 \mathrm{ft}$. of Dr. Linfoot's design be required to separate close images in densely populated Milky Way clouds, it could easily be obtained at the cost of a somewhat reduced angular field. In Prof. Plaskett's view, however, the overriding requirement is the resolution of the tremor disks of adjacent stars on the photographic plate, and the optimum focal length is set by this consideration. Mr. F. Hoyle welcomed the proposed telescope on the ground that if a large instrument could enable astronomy to contribute one really new idea to physics, the result might amply repay the initial cost. Mr. G. M. Sisson, of Messrs. Sir Howard Grubb, Parsons and Co., welcomed Mr. Hargreaves's design as a practical solution of the major difficulties, but deprecated the use of roller bearings, which deform seriously under load. He put in a plea for the modified English yoke mounting, especially if spectroscopic equipment is to add to the length of the tube, and hoped that the virtues of the Wright camera and of the Gaviola test would not be overlooked.

A lively discussion ensued on the practicability of various new materials for different components of the telescope. On a plea for high conductivity as well as low expansion for the mirror, even speculum metal was proposed, in view of the fact that vacuum evaporation has now removed the bogy of tarnishing. Technical difficulties of casting speculum metal, however, led another speaker to urge the superiority of a low-expansion cast iron such as 'Minvar'. Low thermal expansion, as Dr. Linfoot pointed out, is not necessary for the Schmidt plate, and the merits of 'Vitaglass' for the purpose were discussed. Transparent plastics, it was agreed, are quite unsuitable for this plate.

The discussion as a whole revealed extensive support for the views put so forcibly by the Astronomer Royal for Scotland in its early stages; the desirability, even the necessity, of a large reflecting telescope in Great Britain was generally conceded. About the details of the design, divergences of opinion became apparent. But in a scheme of this sort, such differences are inevitable and are to be welcomed; there will be time to thrash out the details when the principle is approved. It is clear that the main body of informed opinion among astronomers in Great Britain is in favour of the - provision of a large telescope here, and there will be general support for the Royal Astronomical Society in its application for funds to construct and erect it. The prestige of the Royal Society has been lent to the scheme, and Sir Robert Robinson, president of the Royal Society, was able to announce at the opening of the Newton Tercentenary celebrations on July 15 that the Chancellor of the Exchequer would ask Parliament to devote a sum of money for the purpose. Details are not yet settled, but it is now agreed that the Isaac Newton Observatory, as it is to be called, should comprise not a mere 72 -in. but a 100-in. reflector together with ancillary equipment.$$
151=
$$ \\ POLYMERS AND POLYMERIZATION}

$O^{N}$ N April 4, the Cherr hal Society held an afternoon symposium to dijotass certain matters relating to high-polymen fomistry in which significant advances haye peen made recently. Six speakers contributed the discussion, but, since the Chemical Socjety fillpublish the papers in full, only a few of the salieht poin 6 f more general interest need be mon ffed here. The first part of the discussion dealt with problems connected with the mechanism of the synthesis of high polymers, and the second part more particularly with the structure of such polymers.

The development of synthetical techniques has revealed at least three distinct ways in which macromolecules may be formed; and the simplest from the purely chemical point of view is the process of polycondensation, exemplified by the combination of dihydric alcohols with dibasic acids to form superesters. These reactions take place by a step-like process. That is, each step is kinetically similar though not identical. The unfortunate fact is that polycondensations cannot be studied in dilute solution, and therefore it is impossible to determine whether molecular size has any effect at all on the magnitude of the kinetic coefficients; nor is it possible to say why in any given case there seems to be a practical upper limit to molecular weight. The next important class is the polymerization of ethylene derivatives in the gas and liquid phases at room temperature and above. Here considerable progress has been made in getting a fairly detailed picture of the mechanism. The third class are those polymerizations which occur at low temperatures, for example, $-80^{\circ} \mathrm{C}$. and are catalysed by substances of the FriedelCrafts type, namely, $\mathrm{BF}_{3}, \mathrm{AlBr}_{3}$, etc. These are confined to hydrocarbons, though there is no doubt that other types of molecule may be made to polymerize in the same way. There may be other types of process, for certain peculiarities so far observed may not come under any of the above headings; but these matters are still too uncertain to be profitably discussed here.

The Friedel-Crafts type of catalysis was discussed by Prof. M. Polanyi. The facts may be summarized briefly by saying that reaction occurs exceedingly rapidly, that products of higher molecular weight are obtained, that the reaction has a high negative temperature-coefficient. It would appear that besides 